\title{
A novel uncultured marine cyanophage lineage with lysogenic potential linked to a putative marine Synechococcus 'relic' prophage
}

José Flores-Uribe ${ }^{1 \star}$, Alon Philosof ${ }^{1,4}$, Itai Sharon ${ }^{2,3}$, Svetlana Fridman ${ }^{1}$, Shirley Larom ${ }^{1}$ and Oded Béjà ${ }^{1 *}$

${ }^{1}$ Faculty of Biology, Technion - Israel Institute of Technology, Haifa 32000 , Israel; ${ }^{2}$ Migal Galilee Research Institute, Kiryat Shmona, 11016, Israel; ${ }^{3}$ Tel Hai College, Upper Galilee 12210, Israel. ${ }^{4}$ Department of Geological and Planetary Sciences, California Institute of Technology, Pasadena, CA 91106, USA.

*Address correspondence to José Flores-Uribe, jfloresu@gmail.com and Oded Béjà, beja@technion.ac.il

Faculty of Biology, Technion - Israel Institute of Technology, Haifa 32000

D.ınning title: A novel uncultured oceanic cyanophage lineage

This article has been accepted for publication and undergone full peer review but has not been through the copyediting, typesetting, pagination and proofreading process which may lead to differences between this version and the Version of Record. Please cite this article as doi: $10.1111 / 1758-2229.12773$ 


\section{Originality-Significance Statement}

Marine cyanobacteria are major contributors to primary production in the ocean. Despite reports of lysogeny in marine cyanobacteria, genomes from lysogenic marine cyanophages have not been reported yet. Using metagenomics assemblies, we recovered complete genomes of a novel uncultured marine cyanophage lineage. Remarkably, the DNA polymerase of these uncultured phages forms a monophyletic clade with the DNA polymerase from a genomic island in Synechococcus WH8016. The genomic island contains a putative relic prophage that does not resemble any known cultured cyanophage but shares several genes with the newly identified cyanophage family. These findings provide both phylogenomic and abundance estimates that are missing from current ecological models of this important group of marine viruses. 


\section{Summary}

Marine cyanobacteria are important contributors to primary production in the ocean and their viruses (cyanophages) affect the ocean microbial communities. Despite reports of lysogeny in marine cyanobacteria, a genome sequence of such temperate cyanophages remains unknown although genomic analysis indicate potential for lysogeny in certain marine cyanophages. Using assemblies from Red Sea and Tara Oceans metagenomes, we recovered genomes of a novel uncultured marine cyanophage lineage, which contain, in addition to common cyanophage genes, a phycobilisome degradation protein NbIA, an integrase and a split DNA polymerase. The DNA polymerase forms a monophyletic clade with a DNA polymerase from a genomic island in Synechococcus WH8016. The island contains a relic prophage that does not resemble any previously reported cyanophage but shares several genes with the newly identified cyanophages reported here. Metagenomic recruitment indicates that the novel cyanophages are widespread, albeit at low abundance. Here we describe a novel potentially lysogenic cyanophage family, their abundance and distribution in the marine environment.

\section{Introduction}

Viruses are the most abundant biological entities on the planet, represent major genetic reservoirs and rewire their hosts metabolism (Breitbart, 2012; Paez-Espino et al., 2016). Cyanophages, viruses infecting cyanobacteria, regulate cyanobacterial communities and influence global nutrient cycles (Puxty et al., 2018). 
In a previous screen of Bacterial Artificial Chromosome (BAC) libraries for photosystem II (PSII) genes, some BACs were identified as originating from cyanobacteria and others resembled known cyanophage genomes (Zeidner et al., 2005). One clone from a Mediterranean Sea sample (BAC21E04) was notable for containing numerous open reading frames (ORFs) with weak or no similarity to sequences reported in Genbank, including both non-redundant and environmental-non-redundant databases, precluding the possibility to assign affiliation to this BAC at the time. Nevertheless, BAC21E04 contains a full length viral-like D1 gene, a partial-length viral-like talC transaldolase gene and a putative ribonucleotide reductase (RNR) class II gene (Zeidner et al., 2005).

Viral infections can be either lytic, resulting in host lysis and virions release, or lysogenic, during which viral genomes integrate into the host chromosomes as prophages and replicate without virion production (Howard-Varona et al., 2017). Lysogeny is widespread amongst marine bacterial isolates (Stopar et al., 2003) and has been shown to occur in natural populations of marine Synechococcus .(McDaniel et al., 2002). Among the benefits and consequences of lysogeny, reviewed elsewhere e.g. (Howard-Varona et al., 2017), it has been proposed to play a role in the protection phages from decay (Breitbart, 2018), contribution to host survival under unfavorable environments by suppression of unneeded metabolic activities (Paul, 2008), and phage-mediated horizontal gene transfer of bacterial DNA (Chen et al., 2018). Although temperate phages infecting either freshwater cyanobacteria Anacystis nidulans (Lee et al., 2006) or marine filamentous cyanobacteria (Ohki and Fujita, 1996) are known, to date prophage induction in marine cyanobacteria has only been studied using natural populations or the cultured Synechococcus GM 9914 (McDaniel and Paul, 2005; McDaniel et al., 2006). 
Synechococcus GM 9914 in a non-axenic culture was shown to produce virus-like particles (VLP) containing single stranded DNA under exposure to either mitomycin C or high-light stress, however the DNA of these VLPS was not sequenced (McDaniel et al., 2006).

Here, we report the identification of a novel, widespread lineage of uncultured cyanophages related to BAC21E04. These cyanophages share properties related to temperate phages and shares synteny with a putative relic prophage in Synechococcus WH8016, suggesting that these uncultured cyanophages are potentially lysogenic.

\section{Results and Discussion}

To increase our knowledge regarding uncultured cyanophages, we examined metagenomic assemblies from the Red Sea (Philosof et al., 2017) and Tara Oceans expedition microbiomes and viromes (Brum et al., 2015; Sunagawa et al., 2015). Four contigs (Supporting Information File 1) of putative viral genomes related to BAC21E04 were identified. These contigs, of about $86 \mathrm{kbp}$ (Supporting Information File 2), contain overlapping terminal regions suggesting that they represent complete, terminally rodundant viral Metagenome Assembled Genomes (MAGs). The four MAGs contained a cyanophage-like ribonucleotide reductase (RNR) class II gene (Supporting Information Figure 1 and Supporting Information File 3), a talC transaldolase gene (Supporting Information Figure 1 and Supporting Information File 4), and an exonuclease (exo). Three MAGs carry tRNA genes, an integrase encoding gene, and a $p s b A$ gene with viral signatures (Sharon et al., 2007) (Supporting Information File 5). Also, detected for the first time in marine cyanophages, genes coding for the phycobilisome degradation protein NbIA 
were found in each of the newly identified cyanophages, and three of the MAGs contain two different copies of $n b / A$. Single $n b l A$ genes have been previously reported in freshwater cyanophages (Gao et al., 2012; Ou et al., 2015) that infect cyanobacteria using phycobilisomes for light harvesting. Phycobilisomes, large extrinsic multisubunit lightharvesting complexes typical of most cyanobacteria, have been found in Synechococcus but not in Prochlorococcus (Dufresne et al., 2003). However, as functional, infectionexpressed phycobilisome pigments biosynthesis genes have been characterized in Prochlorococcus cyanophages (Dammeyer et al., 2008) we cannot assume that the putative host of these nblA carrying cyanophages is Synechococcus. The terminase large subunit (TerL), portal, major capsid protein (MCP), and putative tail proteins were identified based on structural predictions. However, no other known phage structural proteins could be detected as protein identity was too low for alignment-based methods to work.

All four MAGs were analyzed using VirFam (Lopes et al., 2014), which classifies phages into Myoviridae, Podoviridae or Siphoviridae according to their neck protein organization. VirFam identified the predicted MCP, Portal and TerL ORFs, however it could not classify them into any of its neck-type categories, suggesting that these cyanophages could belong to a new lineage (Supporting Information File 6).

Due to the lack of good alignments for the traditionally used "phage marker genes" such as portal, MCP or terL protein we employed a strategy similar to the one used by (Rohwer and Edwards, 2002; Mizuno et al., 2013) for the classification of phages based on total shared core pangenome. The gene content of the MAGs and 82 genomes from cyanophages infecting Synechococcus and Prochlorococcus (Supporting Information File 7) was analyzed identifying 14,564 proteins that cluster into 2,376 orthologs. Analysis of 
the shared orthologs between viruses allowed to delineate four clusters of cyanophages corresponding to the viral families Myoviridae, Podoviridae, Siphoviridae and one composed exclusively of the MAGs from this study (Fig. 1a). A similar pattern was achieved when genomic nucleotide distances were used for clustering (Supporting Information Figure 2). The clustering results suggested that the MAGs presented here belong to a new cyanophage lineage whose closest relatives are the unclassified freshwater Synechococcus cyanophage S-EIV1 (50\% shared core proteome) and the putative siphovirus S-SKS1 (20\% shared core proteome). In our core proteome analysis, S-SKS1 clusters among the Myoviridae group, such re-classification has also been reported before (Roux et al., 2016).

Metagenomic recruitment of the Tara Oceans metagenomic reads into the MAGs was used to determine their coverage (Supporting Information Figure 3), presenceabsence of their genes (Supporting Information Figure 4) as well as abundance estimation in the Tara Oceans datasets (Supporting Information File 8). The mapping results indicated the presence $(>0.01 \%$ relative abundance) of these cyanophages in 36 samples from 16 stations with an average of $0.07 \%$ (Fig. 1c). A viral fraction $(<0.2 \mu \mathrm{m})$ sample from the South Atlantic (TARA_076) had the highest abundance of the new cyanophages $(0.39 \%)$, suggesting the presence of cell-free virions from the new family in the environment. To assess if the viral fraction samples where the MAGs were recruited had cyanobacterial contamination, we estimated cyanobacterial abundance in those samples. Cyanobacterial abundance was estimated using pseudo-mapping with Salmon (v0.12.0alpha) and a collection of cytochrome b6 (petB) sequences (Supporting Information File 9). petB has been used as a marker gene for marine cyanobacteria metagenomic abundance 
estimation (Farrant et al., 2016). Salmon results showed no detection of petB on any of the virome samples, indicating that cyanobacterial contamination in these samples is improbable. However, 24 of the 36 samples where the MAGs were detected correspond to the bacterial fraction $(0.2-3.0 \mu \mathrm{m})$ probably indicating ongoing infections during sampling (Philosof et al., 2017).

The new cyanophages also encode a split DNA polymerase, which forms a new cluster within the T7-like cyanophage DNA polymerase family (Fig. 1b). Unexpectedly, a split DNA polymerase from Synechococcus WH8016 also clustered with the new phage family (Supporting Information File 10). Examination of the vicinity of the DNA polymerase genes in the genome of WH8016 revealed the existence of a previously uncharacterized genomic island of approximately $70 \mathrm{kbp}$ (Fig. 2). The genomic island is delimited by a tRNA-Pro and a tRNA-Arg and contains several genes (such as split DNA polymerase genes, integrase, phage/plasmid related protein; Fig. 2) in synteny that exhibit similarity with ORFs from the new viral lineage (Fig. 3).

Neither production of viral particles nor expression of genes inside the genomic island could be detected, with TEM and qPCR respectively, after exposure of WH8016 to mitomycin $\mathrm{C}$ at $5 \mu \mathrm{g} / \mathrm{mL}$. Although the concentration of mitomycin $\mathrm{C}$ employed in the assay was five times higher than previous reported ones for the marine environment $(1 \mu \mathrm{g} / \mathrm{mL})$ (Paul and Weinbauer, 2010; Knowles et al., 2017). Moreover, we also tried induction of WH8016 using heat-shock, light deprivation, or co-cultivation with other marine cyanobacteria (see Supporting Information experimental procedures) without detection of lysis plaques. 
Prophages can also be induced by exposure to different environmental stimuli as pollutants, metals, sunlight, UV irradiation, nutrient deficiency, or pressure changes (Jiang and Paul, 1994; Cochran et al., 1998; McDaniel et al., 2002; Aertsen et al., 2004; Lee et al., 2006). Additionally, induction of some lysogenic freshwater cyanophages in laboratory conditions requires the use of mitomycin C at $20 \mu \mathrm{g} / \mathrm{mL}$ (Dillon and Parry, 2008) and therefore the mitomycin $\mathrm{C}$ concentration and the environmental stressors used in this study might not have been enough to produce a SOS response in WH8016.

Prophages can undergo "domestication" processes driven by their hosts looking to select for beneficial viral traits while losing genes like those encoding structural and lysis components (Bobay et al., 2014; Howard-Varona et al., 2017). These can eventually result in relic prophages and genomic islands that facilitate chromosomal insertions while providing resistance to other phages (Schwartz and Lindell, 2017) and could influence the expression of neighboring genes (Howard-Varona et al., 2017). Furthermore, the variety of bacterial mobile genetic elements include several examples of entities which can only be mobilized by a helper phage. These entities include phage-inducible chromosomal islands (PICIs) and phage-inducible chromosomal island-like elements (PLEs) (Fillol-Salom et al., 2018). PICls and PLEs have sizes of up to $19 \mathrm{~kb}$, do not possess identifiable regulatory, replication or packaging modules and require the help of an additional phage to excise from the bacterial chromosome (Fillol-Salom et al., 2018). Therefore, under the conditions of our assays and the lack of recognizable viral structural proteins, we conclude that the genomic island in Synechococcus WH8016 represents either a non-mitomycin C inducible phage, an element which requires the help of another phage to mobilize or a putative 'relic' prophage that lost the ability to enter into lytic cycle. 
Based on our observations, we suggest that the newly identified marine phages form a new, potentially lysogenic, cyanophage family related to the newly characterized 'relic' prophage in Synechococcus WH8016. The identification of putative lysogenic SAR11 phages (Zhao et al., 2018) and other recent studies suggest that lysogeny in the marine environment could be implicated in ecological processes on broad scales (Knowles et al., 2016; Wigington et al., 2016; Knowles et al., 2017).

Although almost half of the sequenced bacteria are lysogens (Touchon et al., 2016), available marine cyanobacterial genomes do not contain complete prophages in them. The lack of recognizable prophages in marine cyanobacterial genomes could be a result of multiple factors among which we can enlist two. First, the relatively low number of cyanobacterial genomes available in public databases when compared to other bacterial phyla (Alvarenga et al., 2017) which makes difficult the use of automated prophage detection tools. Second, in accordance to what has been proposed for the recently described temperate pelagiphages (Zhao et al., 2018), many marine cyanobacteria possess a small cell size and streamlined genome architecture (Larsson et al., 2011) which could impose an additional cost to carry prophages (Zhao et al., 2018) and is associated with a low proportion of prophages (Touchon, 2016). A partial prophage found in environmental Prochlorococcus populations (Malmstrom et al., 2013) and a relic prophage identified in marine Synechococcus RS9917 (Sullivan et al., 2009) could be examples of unstable integrations of prophages in marine cyanobacteria.

Along with the abundance estimates for the novel lineage of cyanophages reported here, we suggest that lysogeny in cyanobacteria might be more widespread than previously thought, adding to the range of life styles that marine cyanophages exhibit. 


\section{Author Contributions}

J.F.-U. and O.B conceived the project. J.F.-U., A.P., I.S., and O.B. performed bioinformatic analyses. J.F.-U., S.F. and S.L. performed mitomycin C experiments. J.F.-U. and O.B. wrote the manuscript with contributions from all authors to data analysis, figure generation, and the final manuscript.

\section{Acknowledgements}

We thank Laurence Garczarek for sharing unpublished data for Synechococcus WH8016, and Curtis Suttle and Caroline Chénard for sharing DNA polymerase sequences. This work was funded by a European Commission (ERC Advanced Grant no. 321647), and the Louis and Lyra Richmond Memorial Chair in Life Sciences (to O.B.).

\section{Conflict of interest}

The authors declare no conflict of interest.

\section{Figure legends}

Figure 1. a) Comparison of Cyanophages genomes clustered according to percentage of shared orthologs. Heatmap representation of protein orthologs shared by cyanophages, scaled between 0 (darker) and 100\% (lighter). Dendrogram tips are colored according to the taxonomic affiliation of the cyanophages using the following key: Myoviridae (purple), 
Podoviridae (blue), Siphoviridae (green), and the MAGs identified in this study (red). Clustering identifies four large groups corresponding to the taxonomic families from the cyanophages (full details in the supporting information). b) Phylogenetic protein tree of DNA Polymerase. The newly identified MAGs and Synechococcus WH 8016 are shown in bold red. Cyanophage and cyanobacteria sequences are shown in red and green, respectively. Split DNA polymerases are marked with an asterisk. The MAGs form a monophyletic clade with a split DNA polymerase from a genomic island in Synechococcus WH8016. c) Distribution and relative abundance of the newly identified cyanophages in Tara Oceans metagenomes. Each red circle is scaled to represent the relative abundance of the MAGs in each station. Black points indicate stations where the MAGs could not be detected $(<0.01 \%)$.

Figure 2. A genomic island in the genome of Synechococcus WH8016. Top: Recruitment of Tara Oceans reads from 331 metagenomes onto Synechococcus WH8016 genome. Each recruited read is drawn according to the position in the genome where it aligned and the nucleotide identity to the recruitment area. Reads are colored according to the sample Inaction of origin, red for virome $(<0.2 \mu \mathrm{m})$ and blue for microbiome $(0.2-3.0 \mu \mathrm{m})$. Bottom: Karlin signature $\left(\delta^{\star}\right)$ difference, the difference in the relative abundance of dinucleotides between a sliding window and the whole sequence (Karlin et al., 1998), was calculated using a window size of $10000 \mathrm{bp}$ and a step size of $5000 \mathrm{bp}$. Colored bars spanning both panels indicate position of some of the shared proteins in synteny with the newly identified cyanophages. 
Figure 3. Synteny plots for the MAGs from the reported viral lineage. Distinctive viral genes, cyanophage marker genes and genes of interest are colored according to the legend. Using tblastx regions of similarity among the phages, BAC21E04 and the genomic island of WH8016 were identified. The synteny plot was obtained using Easyfig (Sullivan et al., 2011) and the origin of the MAGs was readjusted for clarity. The figure was completed using the open-source vector graphics editor Inkscape (available from http://inkscape.org/).

Supporting Information Figure 1. Phylogenetic protein trees: Transaldolase and RNR. The newly identified contigs and Synechococcus WH8016 are shown in bold. Cyanophage and cyanobacteria sequences are shown in red and green, respectively.

Supporting Information Figure 2. Clustering of genomic distance among cyanophages infecting Synechococcus and Prochlorococcus using Mash (Ondov et al., 2016). The heat maps illustrate the pairwise similarity as genomic distance between genomes, scaled between 0 (darker) and 1 (lighter). Genome groups are colored in the dendrogram according to the taxonomic group of the cyanophages using the following key: Myoviridae (purple), Podoviridae (blue), Siphoviridae (green), and the MAGs identified in this study (red).

Supporting Information Figure 3. Nucleotide level MAGs Tara Oceans coverage. The coverage of each nucleotide position from the MAGs in the Tara Oceans metagenomes 
was recovered from bowtie2 mappings. On top of each plot a schematic representation of the ORFs genomic maps for each MAG is presented, colored as indicated in the legend.

Supporting Information Figure 4. Anvi'o detection of the MAGs ORFs in the Tara Oceans metagenomes. In box-plots detection values for each ORF in samples with at least $75 \%$ mean MAG detection. Boxes colored according to the sample fraction. Mean $(\bar{x})$ and median (M) displayed next to the corresponding sample.

\section{Supporting Information Legends}

Supporting Information File 1. Sequences in FASTA format for the MAGs identified in the study.

Supporting Information File 2. Genometrics of the MAGs. Properties of the MAGs identified in the study: Length, \%GC content, Number of Open Reading Frames, Position of Open Reading Frames of Interest

c'spporting Information File 3. Amino acid alignments in FASTA format used for the RNR phylogenetic tree.

Supporting Information File 4. Amino acid alignments in FASTA format used for the TalC phylogenetic tree. 
Supporting Information File 5. Annotation table. List of the ORFs and tRNAs identified in the MAGs with putative annotation, method of annotation, DNA sequence and amino acid sequence.

Supporting Information File 6. VirFam results for the classification of the MAGs.

Supporting Information File 7. Cyanophages used for cluster comparisons. List of 86 cyanophage genomes used in the cluster comparison analysis, contains 82 sequenced cyanophages deposited at NCBI GenBank and the 4 MAGs from the study.

Supporting Information File 8. Coverage, detection, and abundance summaries calculated with Anvi'o of the MAGs across the Tara Oceans metagenomes.

Supporting Information File 9. Accessions of the petB sequences used for the cyanobacterial abundance estimation.

Supporting Information File 10. Amino acid alignments in FASTA format used for the DNAPol phylogenetic tree.

Supporting Information File 11. Primers used for qPCR detection of genes inside the genomic island of WH8016. 


\section{References}

Aertsen, A., Van Houdt, R., Vanoirbeek, K., and Michiels, C.W. (2004) An SOS response induced by high pressure in Escherichia coli. J Bacteriol 186: 6133-6141.

Alvarenga, D.O., Fiore, M.F., and Varani, A.M. (2017) A Metagenomic Approach to Cyanobacterial Genomics. Front Microbiol 8: 809.

Bobay, L.M., Touchon, M., and Rocha, E.P. (2014) Pervasive domestication of defective prophages by bacteria. Proc Natl Acad Sci U S A 111: 12127-12132.

Breitbart, M. (2012) Marine Viruses: Truth or Dare. Ann Rev Mar Sci 4: 425-448.

Brum, J., Ignacio-Espinoza, J.C., Roux, S., Doulcier, G., Acinas, S.G., Alberti, A. et al. (2015) Patterns and ecological drivers of ocean viral communities. Science 348: 1261498.

Chen, J., Quiles-Puchalt, N., Chiang, Y.N., Bacigalupe, R., Fillol-Salom, A., Chee, M.S.J. et al. (2018) Genome hypermobility by lateral transduction. Science 362: 207-212.

Cochran, P.K., Kellogg, C.A., and Paul, J.H. (1998) Prophage induction of indigenous marine lysogenic bacteria by environmental pollutants. Mar Ecol Prog Ser 164: 125-133.

Dammeyer, T., Bagby, S.C., Sullivan, M.B., Chisholm, S.W., and Frankenberg-Dinkel, N. (2008) Efficient phage-mediated pigment biosynthesis in oceanic cyanobacteria. Curr Biol 18: 442-448.

Dillon, A., and Parry, J.D. (2008) Characterization of temperate cyanophages active against freshwater phycocyanin - rich Synechococcus species. Freshwater Biol 53: 1253-1261.

Dufresne, A., Salanoubat, M., Partensky, F., Artiguenave, F., Axmann, I.M., Barbe, V. et al. (2003) Genome sequence of the cyanobacterium Prochlorococcus marinus SS120, a nearly minimal oxyphototrophic genome. Proc Natl Acad Sci U S A 100: 10020-10025.

Farrant, G.K., Dore, H., Cornejo-Castillo, F.M., Partensky, F., Ratin, M., Ostrowski, M. et al. (2016) Delineating ecologically significant taxonomic units from global patterns of marine picocyanobacteria. Proc Natl Acad Sci U S A 113: E3365-3374.

Fillol-Salom, A., Martinez-Rubio, R., Abdulrahman, R.F., Chen, J., Davies, R., and Penades, J.R. (2018) Phage-inducible chromosomal islands are ubiquitous within the bacterial universe. ISME J 12: 2114-2128.

Gao, E.B., Gui, J.F., and Zhang, Q.Y. (2012) A novel cyanophage with a cyanobacterial nonbleaching protein A gene in the genome. J Virol 86: 236-245.

Howard-Varona, C., Hargreaves, K.R., Abedon, S.T., and Sullivan, M.B. (2017) Lysogeny in nature: mechanisms, impact and ecology of temperate phages. ISME J 11: 1511-1520.

Jiang, S.C., and Paul, J.H. (1994) Seasonal and diel abundance of viruses and occurence of lysogeny/bacteriocinogeny in the marine environment. Mar Ecol Prog Ser 104: 163172.

Karlin, S., Campbell, A.M., and Mrazek, J. (1998) Comparative DNA analysis across diverse genomes. Annu Rev Genet 32: 185-225.

Knowles, B., Bailey, B., Boling, L., Breitbart, M., Cobian-Guemes, A., Del Campo, J. et al. (2017) Variability and host density independence in inductions-based estimates of environmental lysogeny. Nat Microbiol 2: 17064.

Knowles, B., Silveira, C.B., Bailey, B.A., Barott, K., Cantu, V.A., Cobian-Guemes, A.G. et al. (2016) Lytic to temperate switching of viral communities. Nature 531: 466-470. 
Larsson, J., Nylander, J.A., and Bergman, B. (2011) Genome fluctuations in cyanobacteria reflect evolutionary, developmental and adaptive traits. BMC Evol Biol 11: 187.

Lee, L.H., Lui, D., Platner, P.J., Hsu, S.F., Chu, T.C., Gaynor, J.J. et al. (2006) Induction of temperate cyanophage AS-1 by heavy metal--copper. BMC Microbiol 6: 17.

Lopes, A., Tavares, P., Petit, M.A., Guerois, R., and Zinn-Justin, S. (2014) Automated classification of tailed bacteriophages according to their neck organization. $B M C$ Genomics 15: 1027.

Malmstrom, R.R., Rodrigue, S., Huang, K., Kelly, L., Kern, S., Thompson, A. et al. (2013) Ecology of uncultured Prochlorococcus clades revealed through single-cell genomics and biogeographic analysis. ISME J 7: 184-198.

McDaniel, L., and Paul, J.H. (2005) Effect of nutrient addition and environmental factors on prophage induction in natural populations of marine synechococcus species. Appl Environ Microbiol 71: 842-850.

McDaniel, L., Houchin, L.A., Williamson, S.J., and Paul, J.H. (2002) Lysogeny in marine Synechococcus populations. Nature 415: 496.

McDaniel, L.D., delaRosa, M., and Paul, J.H. (2006) Temperate and lytic cyanophages from the Gulf of Mexico. J. Mar. Biol. Ass. U.K. 86: 517-527.

Mizuno, C.M., Rodriguez-Valera, F., Kimes, N.E., and Ghai, R. (2013) Expanding the marine virosphere using metagenomics. PLoS Genet 9: e1003987.

Ohki, K., and Fujita, Y. (1996) Occurrence of a temperate cyanophage lysogenizing the marine cyanophyte Phormidium persicinum. J Phycol 32: 365-370.

Ondov, B.D., Treangen, T.J., Melsted, P., Mallonee, A.B., Bergman, N.H., Koren, S., and Phillippy, A.M. (2016) Mash: fast genome and metagenome distance estimation using MinHash. Genome Biol 17: 132.

Ou, T., Gao, X.C., Li, S.H., and Zhang, Q.Y. (2015) Genome analysis and gene nblA identification of Microcystis aeruginosa myovirus (MaMV-DC) reveal the evidence for horizontal gene transfer events between cyanomyovirus and host.J Gen Virol 96: 3681-3697.

Paez-Espino, D., Eloe-Fadrosh, E.A., Pavlopoulos, G.A., Thomas, A.D., Huntemann, M., Mikhailova, N. et al. (2016) Uncovering Earth's Virome. Nature 536: 425-430

Paul, J.H. (2008) Prophages in marine bacteria: dangerous molecular time bombs or the key to survival in the seas? ISME J 2: 579-589.

Paul, J.H., and Weinbauer, M. (2010) Detection of lysogeny in marine environments. Man. Aquat. Viral Ecol. 4: 30-33.

Philosof, A., Yutin, N., Flores-Uribe, J., Sharon, I., Koonin, E.V., and Béjà, O. (2017) Novel abundant oceanic viruses of uncultured marine group II euryarchaeota. Curr Biol 27: 1362-1368.

Puxty, R.J., Evans, D.J., Millard, A.D., and Scanlan, D.J. (2018) Energy limitation of cyanophage development: implications for marine carbon cycling. ISME J 12: 1273-1286.

Rohwer, F., and Edwards, R. (2002) The Phage Proteomic Tree: a genome-based taxonomy for phage. J Bacteriol 184: 4529-4535.

Roux, S., Brum, J.R., Dutilh, B.E., Sunagawa, S., Duhaime, M.B., Loy, A. et al. (2016) Ecogenomics and potential biogeochemical impacts of globally abundant ocean viruses. Nature 537: 689-693. 
Schwartz, D.A., and Lindell, D. (2017) Genetic hurdles limit the arms race between Prochlorococcus and the T7-like podoviruses infecting them. ISME J 11: 1836-1851.

Sharon, I., Tzahor, S., Williamson, S., Shmoish, M., Man-Aharonovich, D., Rusch, D.B. et al. (2007) Viral photosynthetic reaction center genes and transcripts in the marine environment. ISME J 1: 492-501.

Stopar, D., Cerne, A., Zigman, M., Poljsak-Prijatelj, M., and Turk, V. (2003) Viral abundance and a high proportion of lysogens suggest that viruses are important members of the microbial community in the Gulf of Trieste. Microb Ecol 46: 249-256.

Sullivan, M.B., Krastins, B., Hughes, J.L., Kelly, L., Chase, M., Sarracino, D., and Chisholm, S.W. (2009) The genome and structural proteome of an ocean siphovirus: a new window into the cyanobacterial 'mobilome'. Environ Microbiol 11: 2935-2951.

Sullivan, M.J., Petty, N.K., and Beatson, S.A. (2011) Easyfig: a genome comparison visualizer. Bioinformatics 27: 1009-1010.

Sunagawa, S., Coelho, L.P., Chaffron, S., Kultima, J.R., Labadie, K., Salazar, G. et al. (2015) Structure and function of the global ocean microbiome. Science 348: 1261359.

Touchon, M., Bernheim, A., and Rocha, E.P. (2016) Genetic and life-history traits associated with the distribution of prophages in bacteria. ISME J 10: 2744-2754.

Wigington, C.H., Sonderegger, D., Brussaard, C.P.D., Buchan, A., Finke, J.F., Fuhrman, J.D. et al. (2016) Re-examination of the relationship between marine virus and microbial cell abundances. 1: 15024.

Zeidner, G., Bielawski, J.P., Shmoish, M., Scanlan, D.J., Sabehi, G., and Béjà, O. (2005) Potential photosynthesis gene recombination between Prochlorococcus \& Synechococcus via viral intermediates. Environ Microbiol 7: 1505-1513.

Zhao, Y., Qin, F., Zhang, R., Giovannoni, S.J., Zhang, Z., Sun, J. et al. (2018) Pelagiphages in the Podoviridae family integrate into host genomes. Environ Microbiol 10.1111/14622920.14487. 


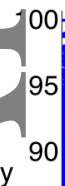 \\ 80}

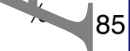

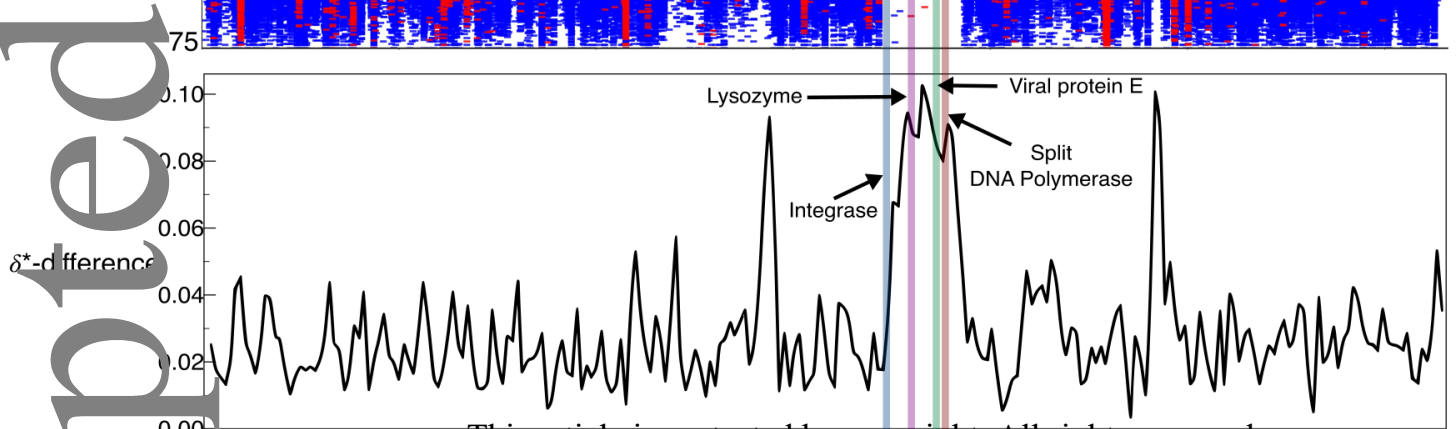

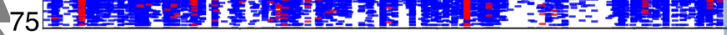
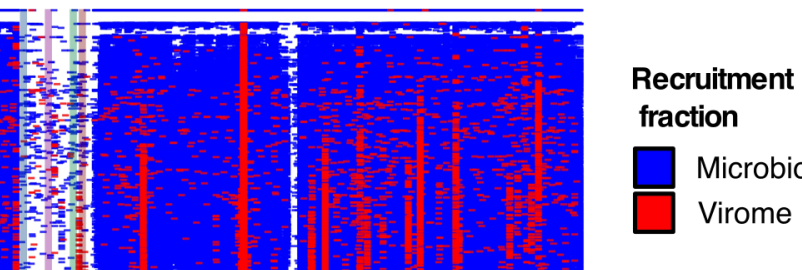

西

Microbiome

Virome 
